\title{
Coronal Hole Oscillations as inferred from SDO/AIA data
}

\author{
Hegde M. ${ }^{\mathrm{a}, \mathrm{b}, *}$, Hiremath K.M. ${ }^{\mathrm{a}}$, Doddamani Vijayakumar H. ${ }^{\mathrm{b}}$ \\ ${ }^{a}$ Indian Institute of Astrophysics, Bangalore 560034, India \\ ${ }^{\mathrm{b}}$ Dept. of Physics, Bangalore University, Bangalore 560056, India
}

Received 22 November 2012; received in revised form 7 March 2014; accepted 1 April 2014

Available online 13 April 2014

\begin{abstract}
With high temporal resolution (12 s) of about two hours duration, data of a coronal hole structure in $171 \AA, 193 \AA$ and $211 \AA$ taken from SDO/AIA images is considered for examination of oscillations. After estimating the total DN counts of a whole coronal hole structure in three wavelength bands, the resulting time series are subjected to FFT and wavelet analysis. Significant periods in all the three wavelength bands are detected that are mainly concentrated around $500 \mathrm{~s}$ as a fundamental mode and its odd (167, 100, 71, 56, 46, 39, 33, 29, 26, 24 s) harmonics. Computed phases in all the three wavelengths band are estimated to be constant.
\end{abstract}

(C) 2014 COSPAR. Published by Elsevier Ltd. All rights reserved.

Keywords: SDO/AIA data; Coronal hole; Flux tube; Oscillations

\section{Introduction}

Apart from global oscillations of the Sun as a whole, other active regions of Sun also oscillate in different periods. Sunspots/ oscillations have been investigated in several works (Kentischer and Mattig, 1995; Lites, 1986; Bogdan, 2000 and references there in). Fleck et al. (1994) studied the solar oscillations with height using He I $10830 \AA$ in quiet solar atmosphere.

Since the launch of EUV telescopes in space, which have very good sensitivity and high spatial and temporal resolutions, coronal oscillations have been studied. As presented by Aschwanden (2003), Table 1 gives a flavor of coronal oscillations as observed in the EUV window.

Detection of oscillations in the chromosphere and corona can help us in understanding the magnetic structure of the solar atmosphere, and may provide valuable insight

\footnotetext{
* Corresponding author at: Indian Institute of Astrophysics, Bangalore 560034, India.

E-mail address: manjunath@iiap.res.in (M. Hegde).
}

into the unresolved coronal heating problem (Tian and Xia, 2008).

Following is a brief history of previous studies on detection of short ( $\sim$ seconds to minutes $)$ and long period ( $\sim$ days) coronal oscillations. The 3-min oscillations are detected above sunspot regions, especially in the transition region (Fludra, 1999; Brynildsen et al., 1999; De Moortel et al., 2002) and, 5-min oscillations are detected in "nonsunspot" loops. Quasi-periodic oscillations in polar plumes were studied by Deforest and Gurman (1998). O'Shea et al. $(2006,2007)$ studied oscillations in plumes, interplumes and coronal holes in the polar regions of the Sun. Periodic oscillations (7-64 $\mathrm{min})$ in coronal bright points are also detected (Ugarte-Urra et al., 2004a,b; Tian et al., 2008). From the globally averaged radio fluxes (at 275, 405, 670, $810,925,1080,1215,1350,1620$ and $1755 \mathrm{MHz})$, Hiremath (2002) detected long period ( $\sim$ days) quiet coronal oscillations.

Coronal holes $(\mathrm{CH})$ are large regions in the solar corona that have low density plasma (Krieger et al., 1973; Neupert and Pizzo, 1974; Nolte et al., 1976; Zirker, 1977; Cranmer, 2009 and references therein; Wang, 2009) with unipolar 
Table 1

Coronal oscillations observed in EUV. Table is taken from Aschwanden (2003).

\begin{tabular}{llll}
\hline Observer & Wavelength $\lambda[\AA]$ & Period P[s] & Instrument \\
\hline Chapman et al. (1972) & $304,315,368$ & 300 & OSO-7 \\
Antonucci et al. (1984) & $554,625,1335$ & 141,117 & Skylab \\
Deforest and Gurman (1998) & 171 & $600-900$ (prop.) & SoHO/EIT \\
Aschwanden et al. (1999) & 171,195 & $276 \pm 25$ & TRACE \\
Nakariakov et al. (1999) & 171 & 256 & TRACE \\
Berghmans and Clette (1999) & 195 & (prop.) & SoHO/EIT \\
De Moortel et al. (2000) & 171 & 256 , 320 (prop.) & TRACE \\
Nakariakov and Ofman (2001) & 171,195 & (prop. waves) & TRACE \\
Robbrecht et al. (2001) & 171,195 & - & TRACE, EIT \\
Schrijver et al. (2002) & 171,195 & $120-1980$ & TRACE \\
Aschwanden et al. (2002) & 171,195 & $282 \pm 93$ (prop.) & TRACE \\
De Moortel et al. (2002a,b) & 171,195 & & TRACE \\
\hline
\end{tabular}

magnetic structures (Harvey and Sheeley, 1979; Harvey et al., 1982) and are connected to interplanetary space. One of the main reasons that $\mathrm{CH}$ are important to study is that they are the primary sites of acceleration of highspeed solar wind that has a significant influence on the Earth's ionosphere, auroras, and on the telecommunication systems (Hiremath and Mandi, 2004 and references there in; Hiremath, 2009; Hiremath and Hegde, 2013 and references there in), etc.

Purpose of this study is to investigate whether a largescale structure like coronal hole oscillates or not. Idea of this study is to examine if large-scale oscillations (rather than small spatial scales) of the coronal hole exist or not. This is equivalent to examining the global oscillations of the sun with small $l$ (degree) modes, such as $l=1$ or $l=2$. As oscillations are in three dimensions, summing of intensity of all the pixels of the coronal hole on the surface is equivalent to examining the oscillations of the entire flux tube along the direction of axis of the coronal hole flux tube. Although small spatial scale oscillations and hence phases of the coronal hole observed at corona cancel along the direction parallel to the photosphere, oscillations of large length scale ( $\sim$ of dimension of visible diameter of the coronal hole) that have same phase at different heights in the corona may exist. Aim of the present study is to probe such oscillations of the coronal hole if exist.

In the present study, using SDO/AIA data, total intensity (DN counts) of the coronal hole structure in three wavelength bands is examined for the oscillations. Structure of this manuscript is as follows. Section 2 introduces data and analysis technique. In Section 3 we present results. In Section 4, with a brief discussion on the nature of detected oscillations, concluding remarks are presented in Section 5.

\section{Data and analysis}

Data is taken from Atmospheric Imaging Assembly (AIA) instrument (Lemen et al., 2012) on board the Solar Dynamics Observatory (SDO). The instrument provides continuous data of the full Sun with four 4096 X 4096 detectors with a resolution of $0.6 \mathrm{arcsec} / \mathrm{pixel}$. AIA observes at ten different wavelength channels in which three are UV-visible channels and seven are EUV channels (94 $\mathrm{A}(\mathrm{Fe}$ XVIII), $131 \AA$ (Fe VIII, XX, XXIII), $171 \AA$ (Fe IX), $193 \AA$ (Fe XII, XXIV), $211 \AA$ (Fe XIV), $304 \AA$ (He II) \& $335 \AA$ (Fe XVI)) respectively. This observational spectral range covers coronal temperature structure from $\sim 0.6$ million ${ }^{\circ} \mathrm{K}$ to $\sim 16$ million ${ }^{\circ} \mathrm{K}$. AIA records near-simultaneous images in each temperature filter with a sustained cadence of currently $12 \mathrm{~s}$. Other details about the instrument can be found in Boerner et al. (2012).

With the help of "Spaceweather.com" website, presence of coronal hole $(\mathrm{CH})$ is confirmed and, from AIA web (http://www.Imsal.com/get aia data/) cut-out service, $\mathrm{CH}$ image is separated for further analysis. This data set contains images observed from 05:00 to 07:00 h UTC on February 08,2011 , in $171 \AA, 193 \AA$ and $211 \AA$ pass bands. Cadence of this data set is $12 \mathrm{~s}$. Further, images with embedded coronal hole data is calibrated using aia_prep.pro. Fig. 1(a) illustrates a typical full disk image of sun taken on February 08, 2011, 05:11:50 UT. Following the method of Hiremath and Hegde (2013), for images of $\mathrm{CH}$, in each wavelength region, threshold intensity (DN counts) for detecting $\mathrm{CH}$ boundary is estimated as follows.

In order to extract physical parameters of $\mathrm{CH}$ from the cut-out images, we use FV interactive FITS file editor (http://heasarc.gsfc.nasa.gov/docs/software/ftools/fv/).

Depending upon shape of the $\mathrm{CH}$, from the FV editor, a circle or an ellipse is drawn covering the whole region of $\mathrm{CH}$ and, average DN counts (intensity) (that is set as a threshold for detecting the boundary) of $\mathrm{CH}$ is computed for detecting the boundary (private communications with Prof. Aschwanden). Similar to Karachik and Pevtsov (2011), for some of the $\mathrm{CH}$ images, threshold is modified to match the visually estimated boundary. This method yields results consistent with the previous intensity histogram methods (Krista and Gallagher, 2009; Krista, 2011; de Toma, 2011 and references there in). In this way, for each wavelengths, typical intensity threshold values for detecting the coronal hole boundary are: (i) for $171 \AA$ 113 DN counts; (ii) for $193 \AA-76$ DN counts and, (iii) for $211 \AA$ - 30 DN counts respectively. Thus, coronal hole pixels are considered if the pixel values have ranges in between 
(a)

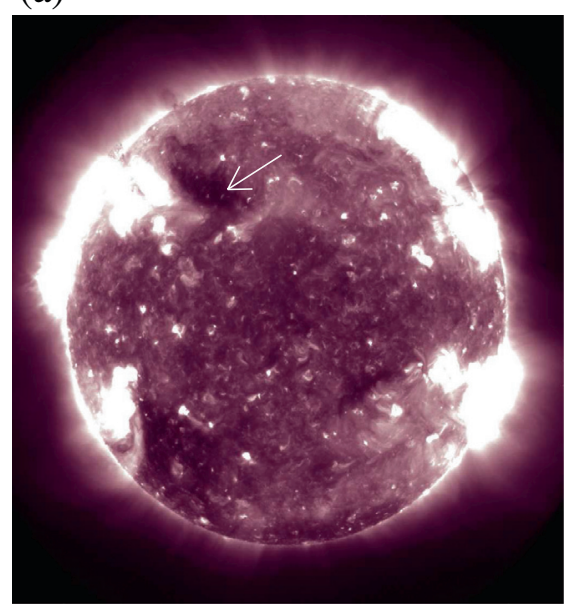

(b)

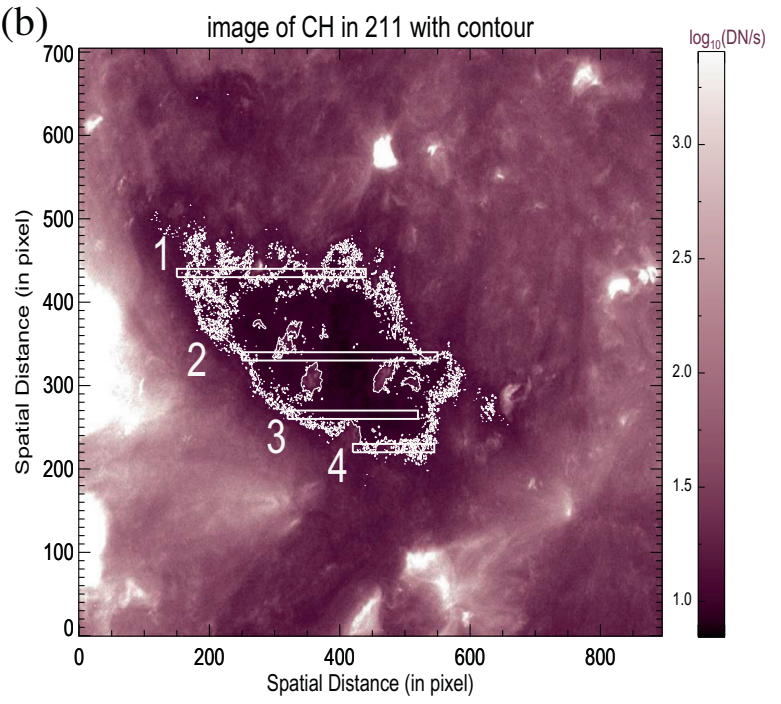

(c)

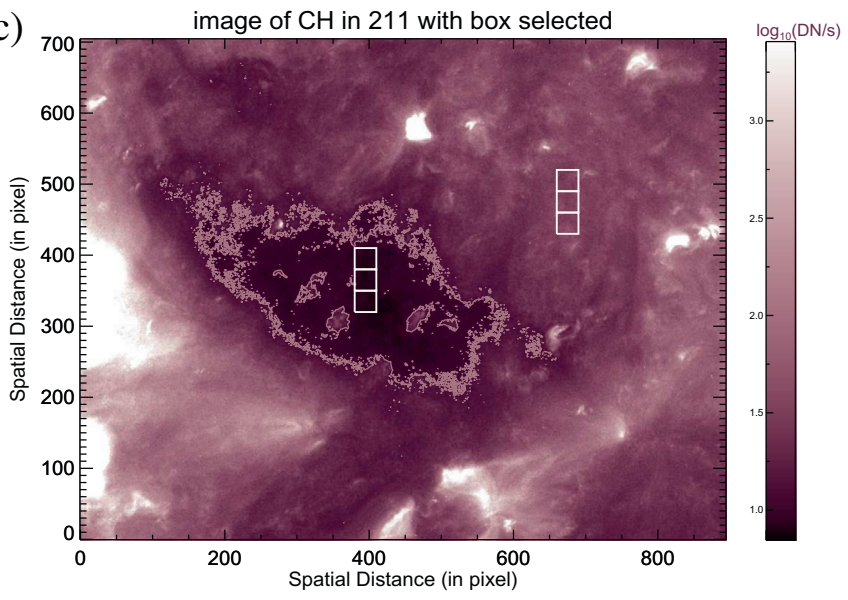

Fig. 1. Fig. 1(a) illustrates full-disk image of sun observed in $211 \AA$ band of SDO/AIA on 08-02-2011, 05:11:50 UT. The arrow in the figure indicates the region of interest for our study. For $211 \AA$, Fig. 1(b) illustrates coronal hole region considered for analysis. Contour is drawn to mark the boundary of the $\mathrm{CH}$ region. In this Fig. 1(b), slits are marked at various positions to plot space-time map as presented in Fig. 2. Fig. 1(c) shows three boxes taken in both $\mathrm{CH}$ and quiet sun (QS) regions to check whether nature of oscillations are same in $\mathrm{CH}$ and QS respectively.

the minimum (10 DN counts, well above the $3 \sigma$ level and the read out noise of $1.1 \mathrm{DN}$ counts, as described in Section 3; where $\sigma$ is standard deviation of DN counts of $\mathrm{CH})$ and the maximum $(\sim$ threshold values that are used for detecting boundary) DN counts. Following Yuan and Nakariakov (2012), see Eq. (3), even if we consider other errors, total noise in each pixel can not exceed the noise of $3 \mathrm{DN}$ counts for all the wavelengths.

Coronal hole (longitude $\sim 23^{\circ}$ East of central meridian and latitude $\sim 31^{\circ}$ North of equator) considered for this study with a boundary detected and marked by white contours is presented in Fig. 1(b). As white color is used only for demarcation of boundary of the coronal hole, one should not confuse with the white color of the original image intensity scale as shown in the colorbar. Total number of pixels and total number of DN counts corresponding to each $\mathrm{CH}$ image with in a marked boundary are computed.
As we consider the derotated images for the analysis, there may be a possibility that artificial saw-tooth like signals from the jerks may be introduced by the spacecraft. If such artifacts are present, one would expect periodic sawtooth like signals in the space-time map. In order to check whether such artifacts are present in the signal, we computed space-time map for different parts of $\mathrm{CH}$ image as follows.

Four positions (with numbering 1, 2, $3 \& 4$ ), two at the boundary and two in the interior of the $\mathrm{CH}$, are considered (Fig. 1(b)) and artificial slits are drawn. With a width of 10 pixels $\left(\approx 6^{\prime \prime}\right)$, following slits with different lengths that cover from the Eastern and Western boundary of $\mathrm{CH}$ are considered. Lengths of four slits are: for 1st slit it is 301 pixels $\left(\approx 180^{\prime \prime}\right)$, 2nd slit it is 301 pixels $\left(\approx 180^{\prime \prime}\right)$, for the $3 \mathrm{rd}$ slit it is 201 pixels $\left(\approx 120^{\prime \prime}\right)$ and for the 4 th slit it is 131 pixels $\left(\approx 78^{\prime \prime}\right)$ respectively. Along lengths of different slits, width of 10 pixel DN counts are averaged for every $12 \mathrm{~s}$ and 
space-time maps are created and are presented in Fig. 2(a). From Fig. 2(a), one can clearly notice that there are no artificial saw-tooth like signals present in space-time maps. Moreover, in case saw-tooth like signal is present, one would get the periods of fundamental mode and all (even and odd) harmonics. Whereas, in the following section, FFT analysis yields the periods that have fundamental and odd harmonics only. Hence, it is unlikely that $\mathrm{CH}$ oscillations that we have detected are due to jerks of the space crafts.
In order to rule out that main contribution for the oscillations of $\mathrm{CH}$ is mainly due to boundary of $\mathrm{CH}$ where there is a transition between the $\mathrm{CH}$ and the oscillating ambient medium, following method is adopted. At each location along the length of artificial slits, grand average and standard deviation $(\sigma)$ of DN counts are computed. After subtracting grand average from the individual average, resulting difference is divided by $\sigma$ for the normalization. In this way one can detect, positive and negative deviations from the grand averages. Off course one should not confuse (a)
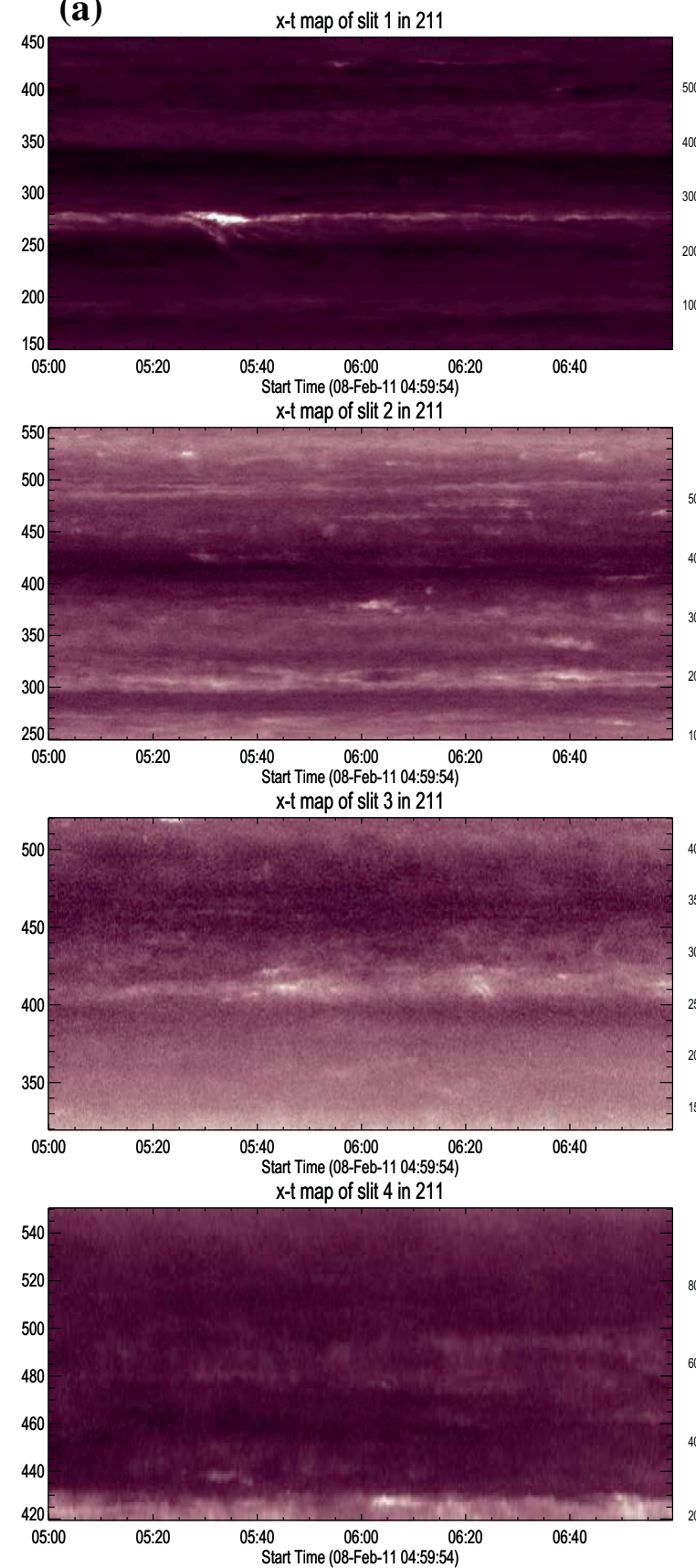

(b)
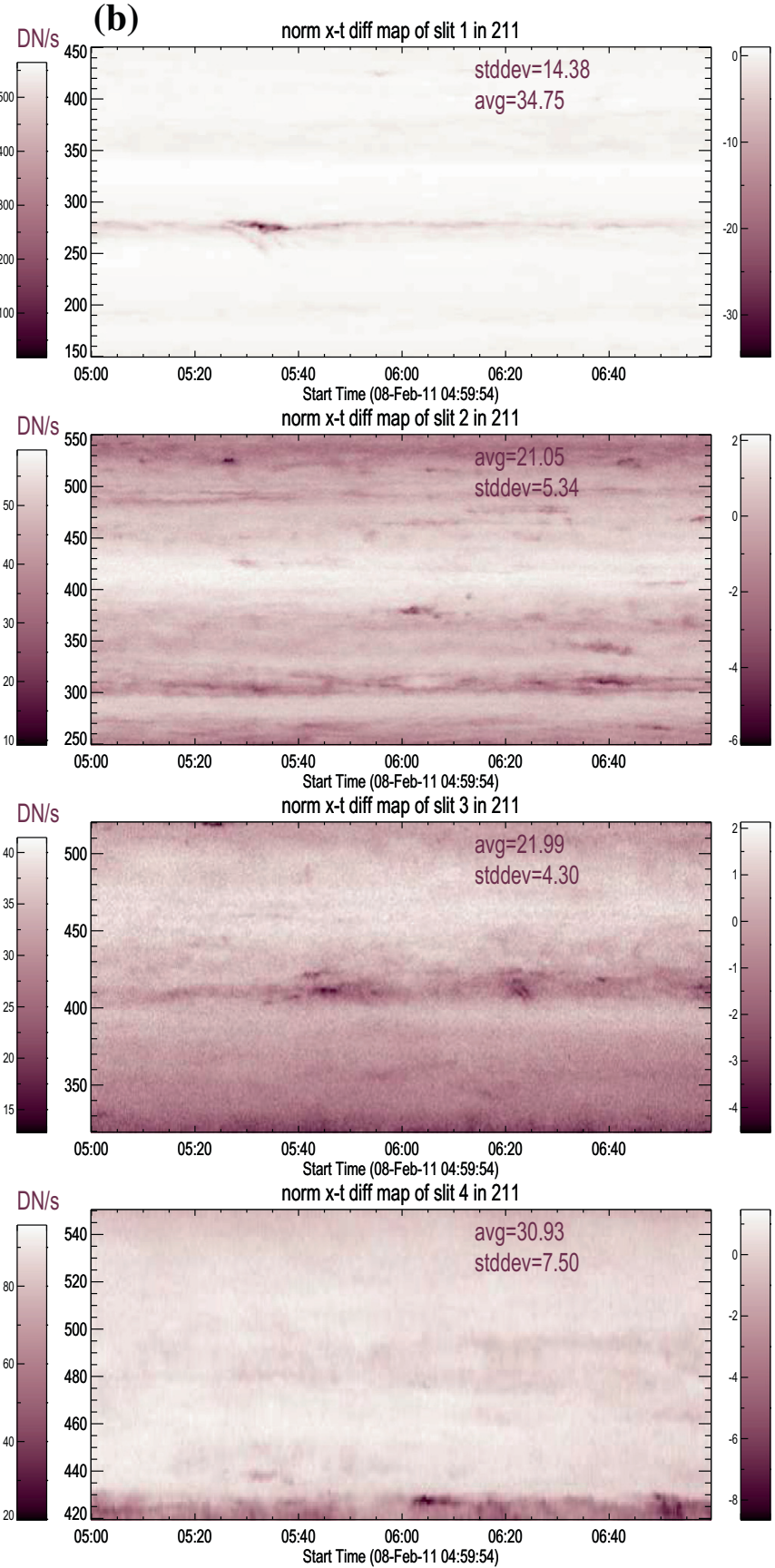

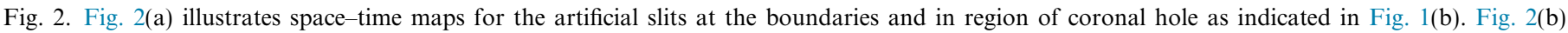

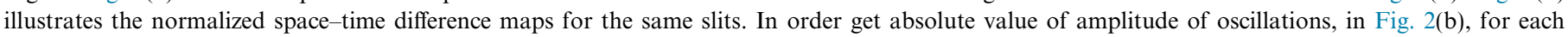
difference map grand average ('avg') and standard deviation ('stddev') of the DN counts are also over plotted. 
with negative oscillations and it means oscillations that have amplitudes smaller than the grand average. In Fig. 2(b), we present such normalized difference space-time maps. For checking the absolute value of amplitude of oscillations, for each map, values of grand average and standard deviation are over plotted in Fig. 2(b). Similar kind of space-time and normalized maps are computed for $171 \AA, 193 \AA$ waveband also and they resemble Fig. 2(a) and (b) respectively. Fig. 2(b) clearly shows dominant oscillation patterns (positive and negative oscillations) inside $\mathrm{CH}$ rather than at the boundaries of $\mathrm{CH}$ indicating that major contribution to the oscillations is coming from inside region of $\mathrm{CH}$ only.

\section{Results}

Once we confirmed that oscillation is real and it is mainly due to $\mathrm{CH}$ only, for the continuous two hour duration of the data set, using the method mentioned above, we estimate total DN counts of the region of coronal hole in three different wavelength bands $(171 \AA, 193 \AA$ and $211 \AA$ ). These total DN counts, for three wavelengths, with respect to time are illustrated in Fig. 3(a) (on the left side, first column).

One can notice that for wavelengths $193 \AA$ and $211 \AA$, during particular periods of time there are sudden bumps in the light curves that are probably due to occurrence of either bright points inside the $\mathrm{CH}$ or sudden input of energy of unknown origin from the surrounding regions of $\mathrm{CH}$. Infact we checked each image during periods of bumpy light curves and found emerging bright points and brightening of whole $\mathrm{CH}$. On the other hand, in between peaks of those bumpy light curves, similar oscillation patterns (as found during normal light curve periods) are also detected. Further, when trend from the original data set is removed, clearly and distinctly, one can notice the oscillation patterns as illustrated in Fig. 3(b). As for removal of trend, we applied a Savitzky-Golay (SAVGOL) smoothing filter (that preserves both low and high peak oscillations; Chen et al., 2004; Press et al., 1992) in order to get a smooth curve (is obtained from IDL SAVGOL routine) that is subtracted from the original light curve.

There are two primary sources (Boerner, private communication) of noise in the images; there is a readout noise of $\sim 1.1 \mathrm{DN}$ in each image, and a shot noise. Shot noise is dominant except in very faint regions like coronal hole. A calculation of flux error analysis in AIA image is done by Yuan and Nakariakov (2012). From this study we find that the detected signal (Fig. 3(b)) of temporal DN count variations in all the three wavelength bands is above the noise level.

Thus, resulting data set in all the three wavelengths band are subjected to FFT and wavelet analysis. Results of power spectrum and phase analysis are illustrated in Fig. 4(a) and (b) respectively. Standard deviations in each power spectrum are computed and periods are detected if power is $\geqslant 1 \sigma$ level. Detected periods are shown in each spectral peak of the FFT plot (Fig. 4(a)). Prominent peaks that have statistically significant periods $(500,167,100,71$, $56,46,39,33,29,26 \mathrm{~s}$ ) are detected.

Further to check whether oscillations of $\mathrm{CH}$ are distinct from QS region, three box regions in $\mathrm{CH}$ and three box regions in QS (Fig. 1(c)) are subjected to FFT analysis. We find similar (if we consider $\mathrm{CH}$ as a whole flux tube) odd harmonics for all the three wavelengths of each box considered in $\mathrm{CH}$. Whereas in QS, as expected, different long periods ( "120 min" and its all the harmonics) and different periods around " $3 \mathrm{~min}$ " are obtained. Probably QS coronal oscillations of long period ( "120 min") are like atmospheric gravity modes of chromosphere as claimed by the previous studies (Dame et al., 1984; Kariyappa et al., 2006) and short period (around "3 min") oscillations are like chromospheric oscillations detected previously (Jensen and Orrall, 1963; Dame et al., 1984; Kariyappa et al., 1994). By using SDO/AIA data, such short period oscillations are also detected in the active region loops (Liu et al., 2011) in the corona. We also computed phases of these oscillations for different boxes in $\mathrm{CH}$ and QS. In $\mathrm{CH}$, phases are constant in all the three wavelengths. Whereas, for all the three wavelengths in QS, phases of long periods, especially around " 120 min" remain approximately constant. Whereas small periods, especially around " $3 \mathrm{~min}$ ", have random phases suggesting propagating waves in the corona. Thus from this periodic analysis, our conclusion is that, with odd harmonics, oscillations of $\mathrm{CH}$ are distinct.

In order to check whether detected periods in $\mathrm{CH}$ as a whole are continuous, we also performed wavelet analysis. In Fig. 5(a)-(c), we illustrate wavelet power spectrum for the $171 \AA, 193 \AA$ and $211 \AA$ wavelengths respectively. One can notice from the wavelet analysis that at the detected periods from the FFT, there is a enhancement of power in the wavelet spectrum. Whereas power concentrated in the hatched region of the wavelet power spectrum is the result of adding zeros to the data set before computing the wavelet spectrum and is not considered to be significant (see the method of obtaining the wavelet transform at the website: http://paos.colorado.edu/research/wavelets/ developed by Torrence and Compo). Thus one has to consider the concentrations of the power above the hatched region as real signals of oscillations.

\section{Discussion}

Before understanding nature (propagating or standing oscillations) of these detected oscillations of $\mathrm{CH}$, we crudely estimate the heights of line formation and the height difference by the following two ways: (i) Having the information of temperature structure from the instrumental response function $\left(\sim 6.5 \times 10^{5} \mathrm{~K}\right.$ for $171 \AA \AA, 1.5 \times 10^{6} \mathrm{~K}$ for $193 \AA$ and $2 \times 10^{6} \mathrm{~K}$ for $211 \AA$ as given by AIA/SDO website) and, assuming that coronal hole and ambient temperature structures are almost same, from the graph (see for example Fig. 2 of Yang et al., 2009) that illustrates 
(a)
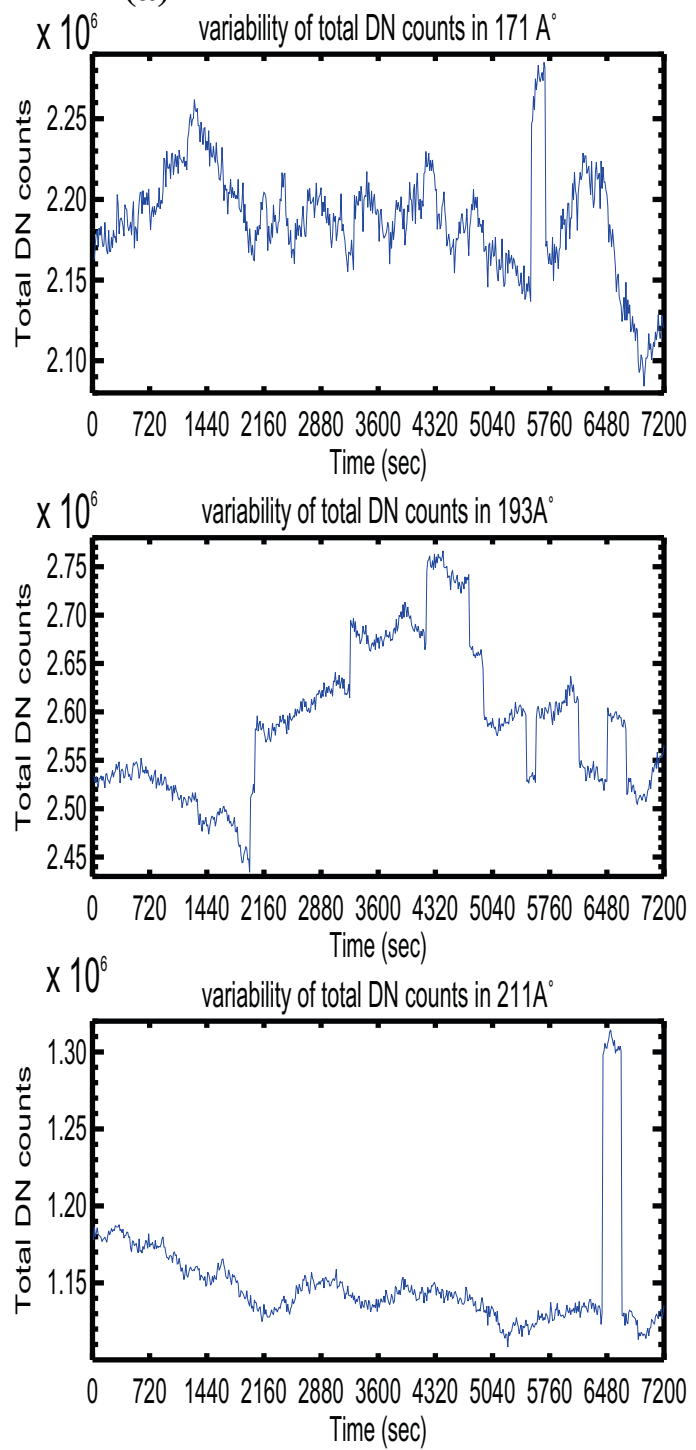

(b)
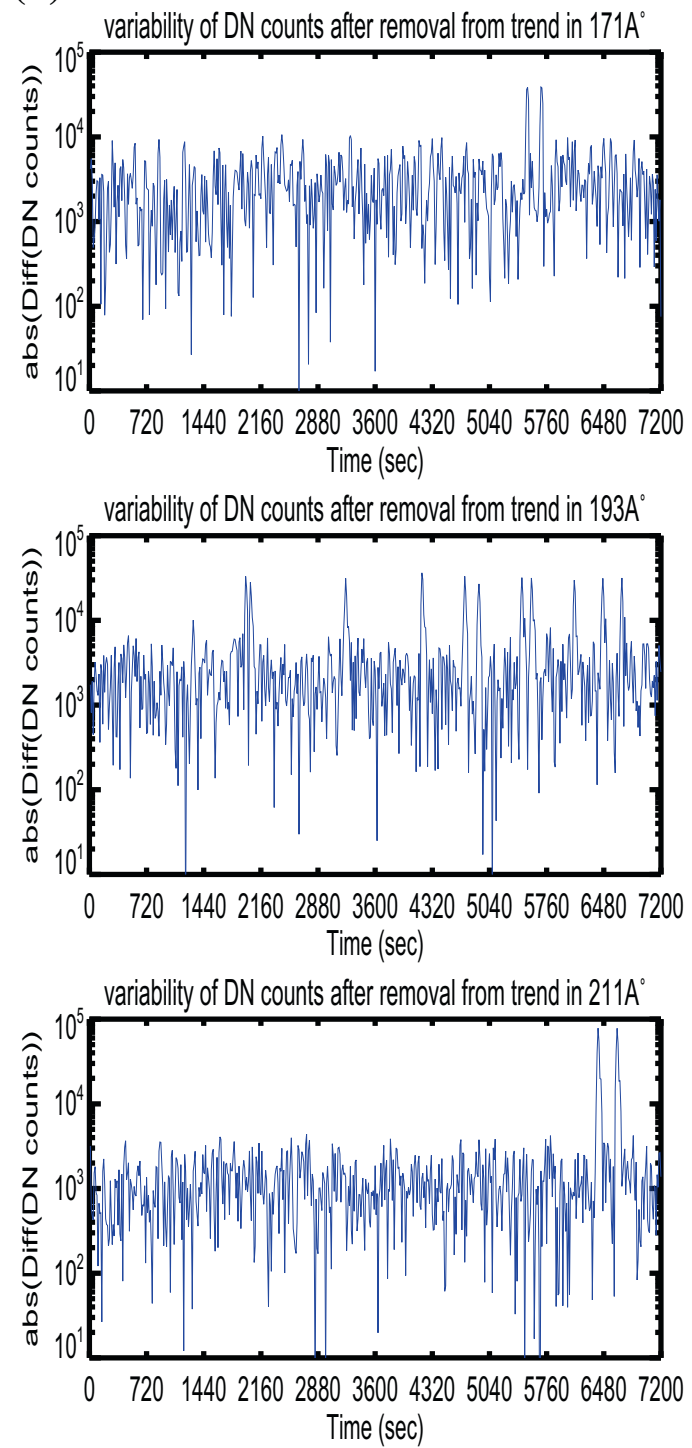

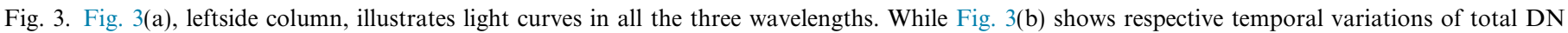
counts of the coronal hole structure after removing the trend.

the relationship between the temperature and heights in the corona, we estimate different heights of line formation for all these three wavelengths. We find that difference in height of line formation between the minimum (at $171 \AA$ ) and the maximum (at $211 \AA$ ) heights is estimated to be $\sim$ $30 \% R_{\odot}$ (where $R_{\odot}$ is the radius of the sun).

(ii) We also estimate the height difference (between one wavelength to another wavelength) in different way as given in the following. If we accept that coronal hole is a magnetic flux tube, increase in height from photosphere to corona results in decrease of ambient pressure of the corona, then $\mathrm{CH}$ flux tube must expand leading to increase in area and decrease in the radiative flux. Infact, we also checked this reasoning by computing average areas and intensity (DN counts) of images for different wavelengths that yield the following results. Average areas of $\mathrm{CH}$ for three wavelengths are: (i) $(0.500 \pm 0.002) \times 10^{20} \mathrm{~cm}^{2}$ for
$171 \AA$, (ii) $(0.980 \pm 0.008) \times 10^{20} \mathrm{~cm}^{2}$ for $193 \AA$ and (iii) $(1.061 \pm 0.010) \times 10^{20} \mathrm{~cm}^{2}$ for $211 \AA$. As for average intensity, computed average DN counts are: (i) $(89.509 \pm 0.011)$ for $171 \AA$, (ii) $(53.852 \pm 0.023)$ for $193 \AA$ and, (iii) $(22.262 \pm 0.009)$ for $211 \AA$.

With this confidence in our reasoning (that with increasing heights area increases and intensity decreases), from Parker's flux tube model (Parker, 1955; also see the details in Hiremath and Lovely, 2007; Hiremath and Lovely, 2012) further we estimate height (from the wavelength $171 \AA$ that has a minimum area to the wavelength $211 \AA$ that has a maximum area) of $\mathrm{CH}$ flux tube as follows.

If we assume $\mathrm{CH}$ as a Parker's flux tube, then strength of magnetic field $B$ varies as $P_{e}^{1 / 2}$ (where $P_{e}$ is an external ambient pressure). Conservation of magnetic flux at different heights yields the relation $B_{1} A_{1}=B_{2} A_{2}$ (where $B_{1}$ and $B_{2}$ are strength of magnetic field structures and $A_{1} \& A_{2}$ 
(a)

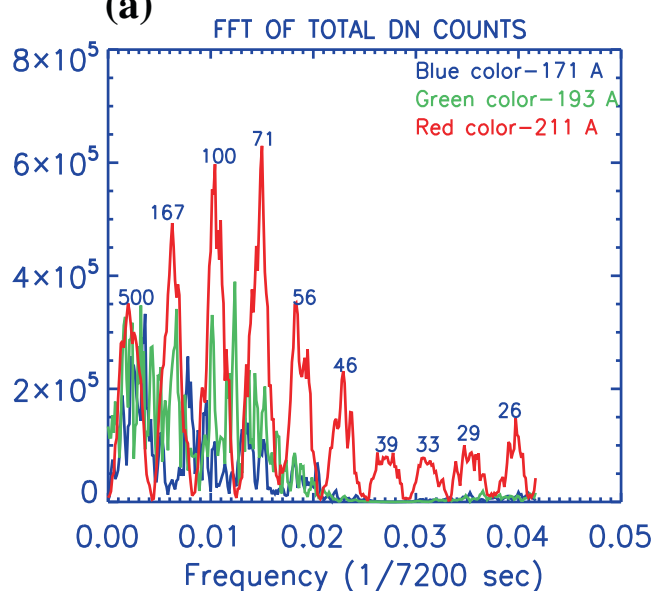

(b)

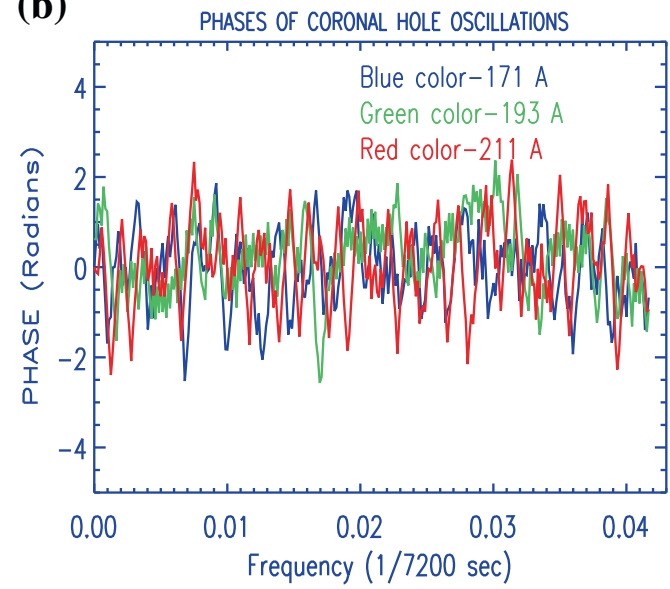

Fig. 4. In three wavelength bands and from the FFT analysis, both the figures on left (4a) and on right (4b) sides illustrate power spectrum and phases of oscillations of the coronal hole.

(a)

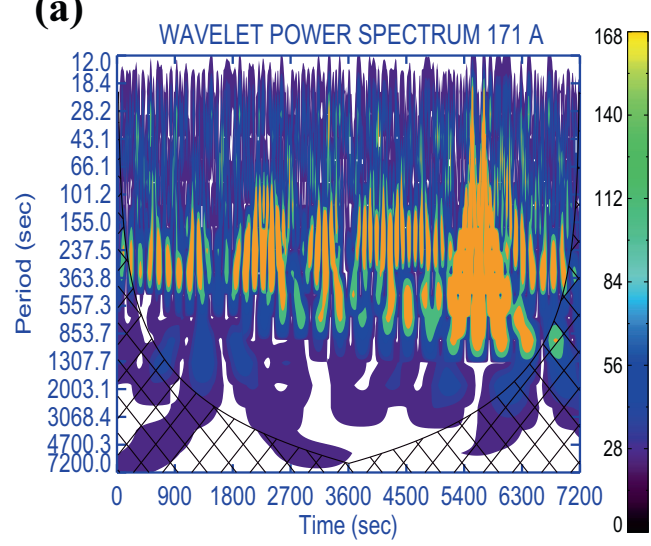

(b)

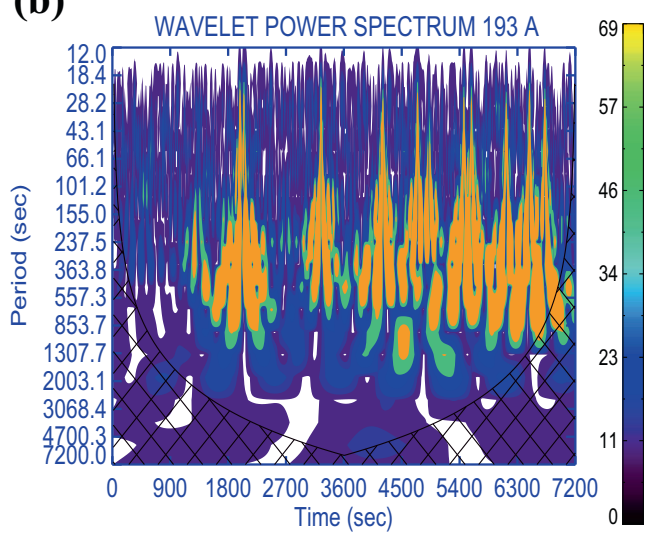

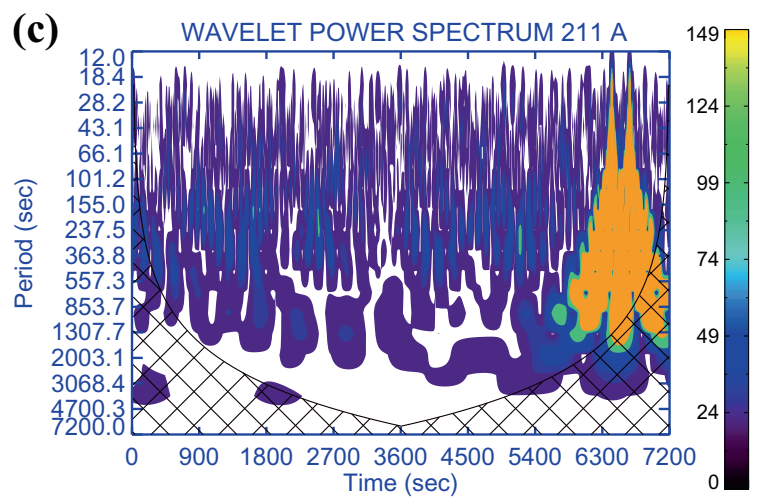

Fig. 5. In three wavelength bands and from the wavelet analysis, all the three figures $(5 \mathrm{a}-\mathrm{c})$ illustrate the wavelet power spectrum (normalized with variance of the power) of oscillations of the coronal hole.

are corresponding areas at two heights) which in turn can be written as $\left(P_{e 1}\right)^{1 / 2} A_{1}=\left(P_{e 2}\right)^{1 / 2} A_{2}$. Further, if we assume that ambient atmosphere is in hydrostatic equilibrium, then $P_{e 2}=P_{e 1} \exp (-z / H)$ (where $z$ is a height variable and $\mathrm{H}$ is pressure scale height). Ultimately conservation of magnetic flux yields formula for height difference $z=2 H \ln \left(A_{2} / A_{1}\right)$ of the flux tube between minimum and the maximum areas respectively.
Hence, by knowing measured areas (of the flux tube at two different wavelengths (or heights)) and the pressure scale height $H$, one can compute the height of flux tube. For the corona and according to Aschwanden (2005), H can be approximated as $\sim 5 \times 10^{9}\left(T_{e} / 1 M K\right) \mathrm{cm}$ (where $T_{e}$ is an external ambient temperature structure). For computation of scale height, average temperatures of all the three wavelengths are considered and the respective height 
difference between the wavelengths $171 \AA$ and $211 \AA$ is estimated to be $\sim(9.75 \pm 0.065) \times 10^{4} \mathrm{kms}$ which is roughly equal to $14 \%$ of the solar radius. One can notice that the height differences estimated by both the methods are almost same $\left(\sim 10^{5} \mathrm{kms}\right)$.

In order to verify whether detected oscillations in $\mathrm{CH}$ are propagating disturbances (traveling waves) or standing oscillations, in all the three wavelength bands, phases of these oscillations are computed and are presented in Fig. 4(b). One can notice from Fig. 4(b) that, in CH, phases for the all three wavelengths remain approximately constant during the period of observation. For constant phases of these oscillation, we have following two possible interpretations that are to be verified by future studies.

If one accepts $\mathrm{CH}$ as a Parker's flux tube, there is a height difference (between $171 \AA$ and $211 \AA$ ) and it is reasonable to conclude that detected oscillations are "standing oscillations". There is also a possibility that the signals observed in different channels could have come from the same height if the plasma is filamented in the horizontal direction (Van Doorsselaere et al., 2008).

\section{Concluding remarks}

To conclude this study, with a high cadence (12 s), coronal hole structure in three (171 $\AA, 193 \AA$ and $211 \AA$ ) wavelengths band as observed by SDO/AIA is considered for examination of oscillations. Boundary of $\mathrm{CH}$ is detected, total DN counts of a whole coronal hole structure in three wavelength bands is estimated. After removing the trend and conforming that oscillation signal is real and it is mainly due to $\mathrm{CH}$, resulting time series are subjected to FFT and wavelet analysis. It is found that in all the three wavelength bands statistically significant periods $(500$, $167,100,71,56,46,39,33,29,26,24 \mathrm{~s})$ are detected. It is interesting to note that, from the detected periods, if we consider $500 \mathrm{~s}$ as the fundamental mode and other detected periods are found to be odd harmonics. For the verification of nature of detected oscillations, phases in all the three wavelengths are computed and it is found that phases of oscillations in three wavelength bands are constant. Possible two interpretations for detected oscillations in $\mathrm{CH}$ are presented. Although temporal oscillations due to whole $\mathrm{CH}$ flux tube are detected, it is also interesting to examine whether spatial scale oscillations of whole $\mathrm{CH}$ for all these three wavelengths exist or not.

\section{Acknowledgments}

Authors are grateful to two anonymous referees for the invaluable comments and suggestions that substantially improved the results and presentation of the manuscript. This work has been carried out under "CAWSES India Phase-II program of Theme 1" sponsored by Indian Space Research Organization (ISRO), Government of India. The AIA data used here is the courtesy of SDO (NASA) and AIA consortium. Hegde, M. is thankful to
SOC (session E2.3, head is Prof. Jean-Claude Vial) of 39th COSPAR Assembly, Mysore, for recommending financial support for attending the meeting. We used the wavelet software developed by $\mathrm{C}$. Torrence and $\mathrm{G}$. Compo and is available at the web site http://paos. colorado.edu/research/wavelets/.

\section{References}

Antonucci, E., Gabriel, A.H., Patchett, B.E., 1984. Oscillations in EUV emission lines during a loop brightening. Sol. Phys. 93, 85-94.

Aschwanden, M.J., 2009. Review of coronal oscillations - an observers view. arXiv:astro-ph/0309505. 9505A.

Aschwanden, M., 2005. Physics of the Solar Corona. Praxis Publishing, UK, Page 69, Eq. 3.1.16.

Aschwanden, M.J., Fletcher, L., Schrijver, C.J., Alexander, D., 1999. Coronal loop oscillations observed with the transition region and coronal explorer. Astrophys. J. 520, 880-894.

Aschwanden, M.J., De Pontieu, B., Schrijver, C.J., Title, A.M., 2002. Transverse oscillations in coronal loops observed with TRACE II. Measurements of geometric and physical parameters. Sol. Phys. 206, 99-132.

Berghmans, D., Clette, F., 1999. Active region EUV transient brightenings - first results by EIT of SOHO JOP80. Sol. Phys. 186, 207-229.

Boerner, P., Edwards, C., Lemen, J.R., et al., 2012. Initial calibration of the atmospheric imaging assembly (AIA) on the solar dynamics observatory (SDO). Sol. Phys. 275, 41-66.

Bogdan, T.J., 2000. Sunspot oscillations: a review (invited review). Sol. Phys. 192, 373-394.

Brynildsen, N., Kjeldseth-Moe, O., Maltby, P., Wilhelm, K., 1999. Nonlinear sunspot transition region oscillations in NOAA 8378. Astrophys. J. 517, 159-162.

Chapman, R.D., Jordan, S.D., Neupert, W.M., Thomas, R.J., 1972. Evidence for the 300-SECOND oscillation from OSO-7 extremeultraviolet observations. Astrophys. J. Lett. 174, 97-99.

Chen, J., Jonsson, P., Tamura, M., Gu, Z., Matsushita, B., Eklundh, B., 2004. A simple method for reconstructing a high-quality NDVI timeseries data set based on the Savitzky-Golay filter. Remote Sens. Environ. 3-4, 321-334.

Cranmer, S.R., 2009. Coronal holes. Living Rev. Sol. Phys. 6, 3.

Dame, L., Gouttebroze, P., Malherbe, J.-M., 1984. Observation and analysis of intensity oscillations in the solar K-line. Astron. Astrophys. $130,331-340$.

Deforest, C.E., Gurman, J.B., 1998. Observation of quasi-periodic compressive waves in solar polar plumes. Astrophys. J. Lett. 501, 217.

De Moortel, I., Ireland, J., Walsh, R.W., 2000. Observation of oscillations in coronal loops. Astron. Astrophys. 355, 23-26.

De Moortel, I., Ireland, J., Hood, A.W., Walsh, R.W., 2002. The detection of $3 \& 5 \mathrm{~min}$ period oscillations in coronal loops. Astron. Astrophys. 387, L13-L16.

De Moortel, I., Ireland, J., Walsh, R.W., Hood, A.W., 2002a. Longitudinal intensity oscillations in coronal loops observed with TRACE I. Overview of measured parameters. Sol. Phys. 209, 61-88.

De Moortel, I., Hood, A.W., Ireland, J., Walsh, R.W., 2002b. Longitudinal intensity oscillations in coronal loops observed with TRACE II. Discussion of measured parameters. Sol. Phys. 209, 89-108.

de Toma, G., 2011. Evolution of coronal holes and implications for highspeed solar wind during the minimum between cycles 23 and 24. Sol. Phys. 274, 195-217.

Fleck, B., Deubner, F.-L., Maier, D., Schmidt, W., 1994. Observations of solar oscillations in He 110830 Å. In: Rabin, D.M., Jefferies, John T., Lindsey, C. (Eds.), Infrared Solar Physics: Proceedings of the 154th Symposium of the IAU; Held in Tucson; Arizona; U.S.A.; 1992. Kluwer Academic Publishers, Dordrecht, p. 65.

Fludra, A., 1999. Intensity oscillations in a sunspot plume. Astron. Astrophys. 344, 75-78. 
Harvey, J.W., Sheeley Jr., N.R., 1979. Coronal holes and solar magnetic fields. Space Sci. Rev. 23, 139-158.

Harvey, K.L., Harvey, J.W., Sheeley Jr., N.R., 1982. Magnetic measurements of coronal holes during 1975-1980. Sol. Phys. 79, $149-160$.

Hiremath, K.M., 2002. Long period global quiet coronal oscillations. ESASP 505, 425-427.

Hiremath, K.M., 2009. Solar forcing on the changing climate. Sun Geosphere 4, 16-21.

Hiremath, K.M., Hegde, M., 2013. Rotation rates of coronal holes and their probable anchoring depths. Astrophys. J. 763, 137.

Hiremath, K.M., Lovely, M.R., 2007. Magnetic flux in the solar convective envelope inferred from initial observations of sunspots. Astrophys. J. 1, 585-590.

Hiremath, K.M., Lovely, M.R., 2012. Toroidal magnetic field structure of the solar convective envelope inferred from the initial observations of the sunspots. New Astron. 4, 392-398.

Hiremath, K.M., Mandi, P.I., 2004. Influence of the solar activity on the Indian monsoon rainfall. NewA 9, 651-662.

Jensen, E., Orrall, F.Q., 1963. Observational study of macroscopic inhomogeneities in the solar atmosphere. IV. Velocity and intensity fluctuations observed in the K line. Astrophys. J. 138, 252.

Karachik, N.V., Pevtsov, A.A., 2011. Solar wind and coronal bright points inside coronal holes. Astrophys. J. 735, 47-52.

Kariyappa, R., Sivaraman, K.R., Anandram, M.N., 1994. Heating of the quiet solar chromosphere. 1: Role of the inner network bright points. Sol. Phys. 151, 243-264.

Kariyappa, R., Dame, L., Hiremath, K.M., 2006. Observational searches for chromospheric g-mode oscillations from calI H-line observations. J. Astrophys. Astron. 27, 327-331.

Kentischer, T.J., Mattig, W., 1995. Oscillations above sunspot umbrae. Astron. Astrophys. 300, 539-548.

Krieger, A.S., Timothy, A.F., Roelof, E.C., 1973. A coronal hole and its identification as the source of a high velocity solar wind stream. Sol. Phys. 29, 505-525.

Krista, L.D., 2011. Ph. D thesis. Trinity College, University of Dublin.

Krista, L.D., Gallagher, P.T., 2009. Automated coronal hole detection using local intensity thresholding techniques. Sol. Phys. 256, 87100.

Lemen, J.R., Title, A.M., Akin, D.J., et al., 2012. The atmospheric imaging assembly (AIA) on the solar dynamics observatory (SDO). Sol. Phys. 275, 17-40.

Lites, B.W., 1986. Photoelectric observations of chromospheric sunspot oscillations. IV - The CA II H line and He I $10830 \AA$. Astrophys. J. 301, 1005-1017.

Liu, W., Title, A.M., Zhao, J., Ofman, L., Schrijver, C.J., Aschwanden, M.J., De Pontieu, B., Tarbell, T.D., 2011. Direct Imaging by SDO/ AIA of quasi-periodic propagating fast mode magnetosonic waves of $2000 \mathrm{~km} / \mathrm{s}$ in the solar corona. Astrophys. J. Lett. 736, 13.
Nakariakov, V.M., Ofman, L., 2001. Determination of the coronal magnetic field by coronal loop oscillations. Astron. Astrophys. 372, $53-56$.

Nakariakov, V.M., Ofman, L., Deluca, E.E., Roberts, B., Davila, J.M., 1999. TRACE observation of damped coronal loop oscillations: implications for coronal heating. Science 285, 862-864.

Neupert, W.M., Pizzo, V., 1974. Solar coronal holes as sources of recurrent geomagnetic disturbances. J. Geophys. Res. 79, 3701.

Nolte, J.T., Krieger, A.S., Timothy, A.F., et al., 1976. Coronal holes as sources of solar wind. Sol. Phys. 46, 303-322.

O’Shea, E., Banerjee, D., Doyle, J.G., 2006. Magnetoacoustic wave propagation in off-limb polar regions. Astron. Astrophys. 452, 10591068.

O'Shea, E., Banerjee, D., Doyle, J.G., 2007. A statistical study of wave propagation in coronal holes. Astron. Astrophys. 463, 713-725.

Parker, E.N., 1955. The formation of sunspots from the solar toroidal field. Astrophys. J. 121, 491.

Press, W.H., Teukolsky, S.A., Vetterling, W.T., Flannery, B.P., 1992. Numerical Recipes in C: The Art of Scientific Computing, second ed. Cambridge University Press.

Robbrecht, E., Verwichte, E., Berghmans, D., Hochedez, J.F., Poedts, S., Nakariakov, V.M., 2001. Slow magnetoacoustic waves in coronal loops: EIT and TRACE. Astron. Astrophys. 370, 591-601.

Schrijver, C.J., Aschwanden, M.J., Title, A.M., 2002. Transverse oscillations in coronal loops observed with TRACE I. An overview of events, movies, and a discussion of common properties and required conditions. Sol. Phys. 206, 69-98.

Tian, H., Xia, L.-D., 2008. Network oscillations at the boundary of an equatorial coronal hole. Astron. Astrophys. 488, 331-337.

Tian, H., Xia, L.-D., Li, S., 2008. Long-period oscillations in solar coronal bright points. Astron. Astrophys. 489, 741-745.

Ugarte-Urra, I., Doyle, J.G., Madjarska, M.S., O’Shea, E., 2004a. Signature of oscillations in coronal bright points. Astron. Astrophys. 418, 313-324.

Ugarte-Urra, I., Doyle, J.G., Nakariakov, V.M., Foley, C.R., 2004b. CDS wide slit time-series of EUV coronal bright points. Astron. Astrophys. 425, 1083-1095.

Van Doorsselaere, T., Brady, C.S., Verwichte, E., Nakariakov, V.M., 2008. Seismological demonstration of perpendicular density structuring in the solar corona. Astron. Astrophys. 491, 9-12.

Wang, Y.-M., 2009. Coronal holes and open magnetic flux. Space Sci. Rev. 144, 383-399.

Yang, S.H., Zhang, J., Jin, C.L., Li, L.P., Duan, H.Y., 2009. Response of the solar atmosphere to magnetic field evolution in a coronal hole region. Astron. Astrophys. 501, 745-753.

Yuan, D., Nakariakov, V.M., 2012. Measuring the apparent phase speed of propagating EUV disturbances. Astron. Astrophys. 543, 9.

Zirker, J.B., 1977. Coronal holes and high-speed wind streams. Rev. Geophys. Space Phys. 15, 257-269. 